\title{
The Protein Kinase 2 Inhibitor CX-4945 Induces Autophagy in Human Cancer Cell Lines
}

\author{
Jiyeon Kim, ${ }^{\ddagger, a}$ Mikyung Park, ${ }^{\dagger, \S, a}$ Byung Jun Ryu, ${ }^{\dagger}$ and Seong Hwan Kim ${ }^{\dagger, *}$ \\ ${ }^{\dagger}$ Laboratory of Translational Therapeutics, Pharmacology Research Center, Korea Research Institute \\ of Chemical Technology, Daejeon 305-600, Korea. ${ }^{*}$ E-mail: hwan@krict.re.kr \\ Department of Biomedical Laboratory Science, School of Medicine, Eulji University, Daejeon 301-746, Korea \\ ${ }^{\S}$ Department of Biotechnology, Translational Research Center for Protein Function Control, \\ College of Life Science and Biotechnology, Yonsei University, Seoul 120-749, Korea \\ Received May 21, 2014, Accepted June 13, 2014
}

\begin{abstract}
Autophagy is a self-digestion process in which intracellular structures are degraded in response to stress. Notably, prolonged autophagy leads to cell death. In this study, we investigated whether CX-4945, an orally available protein kinase 2 (CK2) inhibitor, induces autophagic cell death in human cervical cancer-derived HeLa cells and in human prostate cancer-derived LNCaP cells. CX-4945 treatment of both cell lines resulted in the formation of autophagosomes, in the conversion of microtubule-associated protein 1 light chain 3 (LC3), and in down-regulation of the Akt-mammalian target of rapamycin (mTOR)-p70 ribosomal protein S6 kinase (S6K) signaling cascade. Thus, pharmacologic inhibition of CK2 by CX-4945 induced autophagic cell death in human cancer cells by down-regulating Akt-mTOR-S6K. These results suggest that autophagy-inducing agents have potential as anti-cancer drugs.
\end{abstract}

Key Words : CK2, CK-4945, Autophagy, Cancer

\section{Introduction}

Autophagy is a cell-survival self-digestion process in which intracellular structures are degraded in response to stress. Autophagy is characterized by the formation of doublemembrane autophagosomes that sequester cytoplasmic material and organelles. These autophagosomes fuse with lysosomes to form autolysosomes in which the degradation of cellular structures occurs. ${ }^{1}$ In many organisms in which cells are undergoing apoptosis, autophagy can remove damaged or surplus organelles. Autophagy maintains homeostasis in organisms; in addition, it provides amino acids and fatty acids for use in protein synthesis and contributes to ATP production through the mitochondrial electron transport system. ${ }^{2,3}$ However, when autophagy is prolonged, it can lead to cell death. As one of several types of programmed cell death, autophagy can occur in normal physiological conditions and is also stimulated by stressful conditions such as oxidative stress, starvation, and treatment with pharmacological agents. ${ }^{4,5}$ These types of stress subsequently induce cell death ${ }^{6}$ and this cell death often involves induction of apoptosis through caspase activation.

Several signaling pathways regulate autophagic cell death in cancer cells. ${ }^{6}$ Among these, the mammalian target of rapamycin (mTOR) kinase pathway is a major negative regulator of autophagy in human cancer cells. mTOR prevents autophagy by maintaining the hyperphosphorylation state of its substrates, but its activity is rapidly shut down in response to stress, which allows for the rapid induction of autophagy. ${ }^{7,8}$

\footnotetext{
${ }^{\mathrm{a}}$ These authors contributed equally to this work.
}

Furthermore, Akt and the p70 ribosomal protein S6 kinase (S6K), which are kinases that are upstream and downstream of mTOR signaling, can also negatively regulate autophagy. ${ }^{9}$

Although autophagy itself has a dual role in terms of having both tumor-promoting and tumor-suppressing effects, induction of autophagy-mediated cell death is an efficient approach to killing cancer cells. ${ }^{10,11}$ Thus, targeted induction of autophagy may have potential as a novel anti-cancer strategy. Indeed, recent studies support the notion that induction of autophagy in tumor cells faced with nutrient or oxygen deprivation can be a potent strategy for anti-cancer agents. ${ }^{12}$

In this scenario, anti-cancer drugs could be used to enhance cancer cell death. Recent studies have demonstrated in several types of cancer cells that many chemotherapy drugs work via inducing or inhibiting autophagy by targeting autophagyassociated signaling molecules, including mTOR. ${ }^{6}$

CX-4945, an ATP-competitive small molecule inhibitor of protein kinase 2 (formerly casein kinase 2, CK2), is orally available and has been developed in human clinical trials. The anticancer activity of CX-4945 was exhibited with the inhibition of CK2-regulated PI3K/Akt signaling pathways in several types of cancers. ${ }^{13,14} \mathrm{CX}-4945$ induces dephosphorylation of Akt (S129, T308 and S473) and it causes cell cycle arrest, blocking of inflammatory cytokine secretion, and consequent apoptotic responses. ${ }^{15-17}$ In addition, we also evaluated the pharmacokinetic characteristics of CX- $4945^{18}$ and the inhibitory effect on TGF- $\beta 1$-medicated epithelial-tomesenchymal transition (EMT) in human lung adenocarcinoma cells. ${ }^{19}$ Based on these findings, we demonstrate that CX-4945 can be used as a potent anti-cancer agent to regulate 
cancer cell proliferation, apoptosis, and autophagy through the inhibitory processes of CK2-mediated PI3K/Akt/mTOR signaling pathway.

\section{Experimental}

Cell Culture. Human cervical carcinoma-derived HeLa cells and human prostate cancer-derived LNCaP cells were cultured in DMEM (HeLa) or RPMI1640 (LNCaP; Hyclone, UT) supplemented with $10 \%$ fetal bovine serum (FBS), 100 $\mathrm{U} / \mathrm{mL}$ of penicillin, and $100 \mu \mathrm{g} / \mathrm{mL}$ of streptomycin in a humidified atmosphere of $5 \% \mathrm{CO}_{2}$ at $37{ }^{\circ} \mathrm{C}$. The culture medium was changed every 3 days.

Cell Viability Assay. HeLa cells were seeded in a 96-well plate at a density of $5 \times 10^{3}$ cells/well. After $24 \mathrm{~h}$, the cells were incubated in serially diluted CX-4945 (Sequoia Research Products, Pangbourne, UK) for 1 to 3 days. Cell viability was measured in triplicate using the Cell Counting Kit- 8 (CCK-8; Dojindo Molecular Technologies, USA) according to the manufacturer's protocol. Absorbance was measured using a Wallac EnVision microplate reader (Perkin Elmer, Finland).

Caspase-3 Activity Assay. HeLa cells were seeded in a 96-well plate at the density of $5 \times 10^{3}$ cells/well. After $24 \mathrm{~h}$, cells were incubated in serially diluted CX-4945 for 1 day. Caspase- 3 activity was determined using the Caspase 3 Activity Kit (Promega, Madison, WI, USA).

Quantification of Autophagy. HeLa GFP-LC3 cells were established as described previously. ${ }^{20}$ HeLa GFP-LC3 cells were seeded in a black and clear 96-well plate (Greiner BioOne, NC) at a density of $1 \times 10^{4}$ cells/well. After $24 \mathrm{~h}$, cells were incubated in serially diluted CX-4945 for 1 day. Cells were fixed with $10 \%$ formalin, washed twice with PBS, stained with $10 \mu \mathrm{g} / \mathrm{mL}$ Hoechst 33342 for $5 \mathrm{~min}$, and washed twice with PBS. The GFP-LC3 spots in the cytoplasm were counted using the 'Spot Counting' program that is part of the Thermo Scientific Cellomics ArrayScan HCS reader (PA). Significance was determined using the Student's $t$-test, and differences were considered significant when $p<0.05$.

Transmission Electron Microscopy (TEM) Analysis. HeLa or LNCaP cells were seeded at a density of $1 \times 10^{5} / \mathrm{ml}$ on $60-\mathrm{mm}^{2}$ culture dishes and treated with CX-4945 (10 $\mu \mathrm{M})$ for $24 \mathrm{~h}$. Harvested cells were fixed with $1 \%$ glutaraldehyde in PBS for $2 \mathrm{~h}$ at $4{ }^{\circ} \mathrm{C}$. After washing with $0.1 \mathrm{M}$ cacodylate buffer containing $0.1 \% \mathrm{CaCl}_{2}$, cells were postfixed with $1 \% \mathrm{OsO}_{4}$ for $1 \mathrm{~h}$ at $4{ }^{\circ} \mathrm{C}$. After dehydration, cells were embedded in Embed-812 (Electron Microscopy Science, PA, USA). Ultrathin sections were cut with a Leica EM UC6 microtome and post-stained with uranyl acetate and lead citrate. Images of the sections were observed using a H-7650 transmission electron microscope (Hitachi, Tokyo, Japan).

Western Blot Analysis. HeLa cells $\left(2 \times 10^{5}\right.$ cells $)$ were incubated with CX-4945 for $24 \mathrm{~h}$ and Western blot analysis was performed. Briefly, cells were homogenized and centrifuged at $10,000 \times \mathrm{g}$ for $15 \mathrm{~min}$. The supernatant was considered the cytoplasmic protein fraction. Denatured proteins were separated on the gels and transferred onto PVDF
(Millipore, Temecula, CA, USA). After incubation with the appropriate antibody, membranes were developed with Pierce SuperSignal West Femto Maximum Sensitivity Substrate (Fisher Scientific, Pittsburgh, PA, USA) and signals were visualized using the LAS-3000 luminescent image analyzer (Fuji Photo Film Co., Ltd., Japan). Primary antibodies against p-mTOR, mTOR, p-S6K, S6K, p-Akt, Akt, and LC3 were purchased from Cell Signaling Biotechnology, Inc. (USA). Actin antibody and secondary antibodies were purchased from Santa Cruz Biotechnology, Inc. (USA).

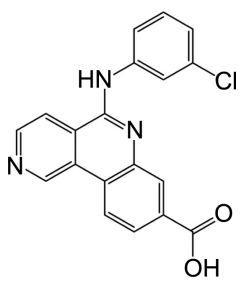

Figure 1. The structure of CX-4945.

(a)

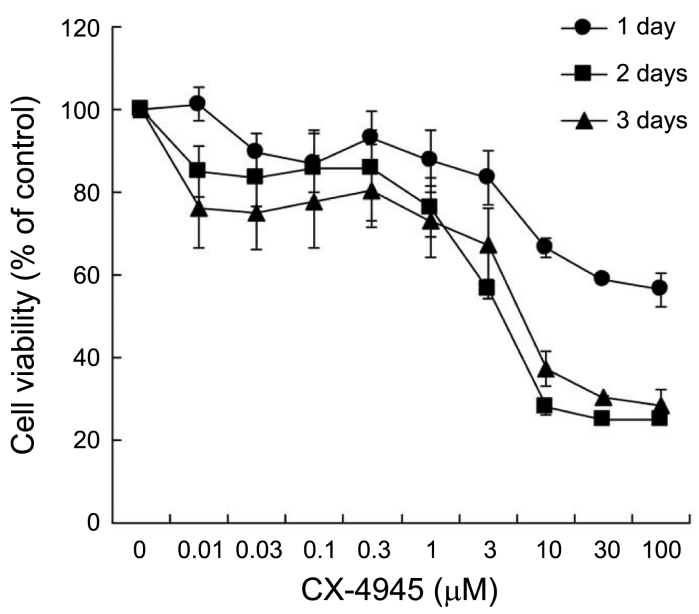

(b)

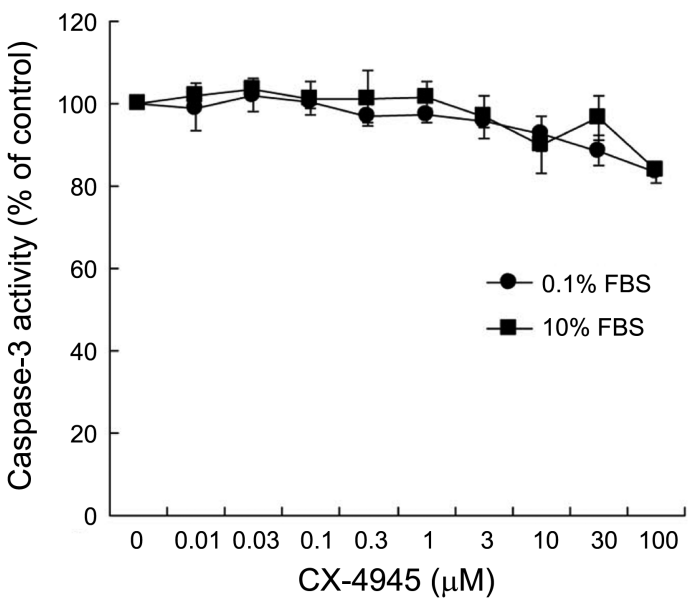

Figure 2. CX-4945 decreased cell viability but did not induce caspase-3. (a) HeLa cells were treated with CX-4945 for 1, 2 and 3 days. Relative cell viability was measured using a CCK-8 assay. (b) HeLa cells were treated with CX-4945 for $24 \mathrm{~h}$ in the presence of culture media containing $0.1 \%$ or $10 \%$ FBS. The relative caspase-3 activity was measured using a caspase-3 activity assay kit. Detailed experimental procedures are described in the Experimental section. All experiments were performed in triplicate. 
LNCaP cells were seeded at a density of $5 \times 10^{5}$ cells $/ \mathrm{ml}$ in a $60-\mathrm{mm}^{2}$ culture dish. After 1 day of culture, cells were serumstarved for $16 \mathrm{~h}$ and treated with vehicle $(0.1 \% \mathrm{DMSO})$ or $10 \mu \mathrm{M}$ CX-4945 for $0.5,1,2,4,6,12$, or $24 \mathrm{~h}$. Western blot analysis was then performed as described above.

\section{Results and Discussion}

CX-4945 (Fig. 1) was synthesized and developed by Cylene Pharmaceuticals (San Diego, CA, USA) as a protein kinase 2 (formerly casein kinase 2; CK2) inhibitor, and clinical trials are ongoing. ${ }^{14}$ Previous studies found that CX4945 has anti-proliferative, anti-angiogenic, and anti-inflammatory effects due to inhibition of CK2-mediated signaling pathways in human cancer cells. ${ }^{15-17}$ Recently our laboratory demonstrated that CX-4945 has good pharmacokinetic profile, shows promise as a potent drug candidate, and blocks the tumor growth factor- $\beta 1$-induced EMT in human adenocarcinoma cells. ${ }^{18,19} \mathrm{CK} 2$ is involved in apoptosis and autophagy via regulation of signaling pathways, including the mTOR pathway. ${ }^{21,22}$ Despite this anti-cancer and anti-EMT activity in several types of human cancers, no studies have investigated CX-4945-mediated autophagic cell death.

The number of autophagosomes in cells can be detected in several ways. In this study, we assessed autophagy using the following methods: we measured the intensity of green fluorescent protein (GFP)-LC3 vacuoles using high-content screening (HCS) analysis; we monitored the conversion of the cytoplasmic form of LC3 (LC3-I, 18-kDa) to the preautophagosomal and autophagosomal membrane-bound form of LC3 (LC3-II, 16-KDa); we assessed the expression levels of autophagy-related proteins using Western blot analysis; and we investigated the formation of autophagosomes by transmission electron microscopy (TEM). These experiments used two cell lines: human cervical cancerderived HeLa cells and human prostate cancer-derived $\mathrm{LNCaP}$ cells.

Before investigating whether CX-4945 induced autophagy, the anti-cancer activity of CX-4945 was evaluated in HeLa cells. CX-4945 decreased cell viability (Fig. 2(a)) but did not affect the activation of caspase-3, the executor of apoptosis (Fig. 2(b)). A novel diquinoline with anti-cancer properties induces caspase-independent autophagic cell death, and down-regulation of CK2 activity facilitates tumor necrosis factor- $\alpha$-mediated chondrocyte death through apoptosis and autophagy. Accordingly, we investigated whether CX-4945 induced autophagy in cancer cells. ${ }^{21,23}$

The effect of CX-4945 on the induction of autophagy was evaluated in HeLa GFP-LC3 cells by visualizing and counting GFP-LC3 spots using the Cellomics Arrayscan reader. Cell image analysis revealed that CX-4945 significantly increased the number of GFP-LC3 spots in HeLa cells in a dose-dependent manner (Fig. 3(a) and 3(b)). To confirm that CX-4945 induced autophagy, the formation of autophagosome in HeLa cells was visualized by TEM (Fig. 3(c)). Since the Akt/mTOR/S6K signaling pathways are involved in autophagy, the levels of phosphorylated Akt, mTOR, and S6K were evaluated. As shown in Figure 3(d), CX-4945 down-regulated Akt, mTOR, and S6K phosphorylation in a dose-dependent manner.

The autophagy-inducing activity of CX-4945 was investigated further in LNCaP cells because we found previously that CX-4945 had anti-androgen receptor activity and an apoptotic effect on human prostate cancer LNCaP cells. ${ }^{24}$ (a)

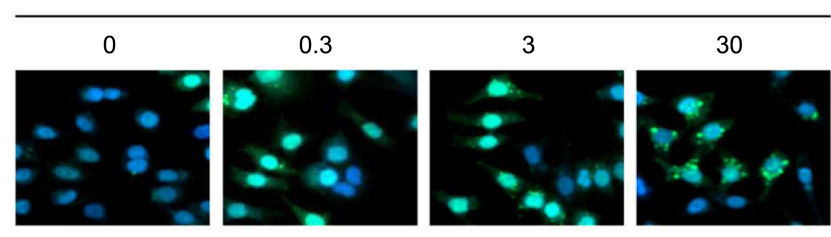

(b)

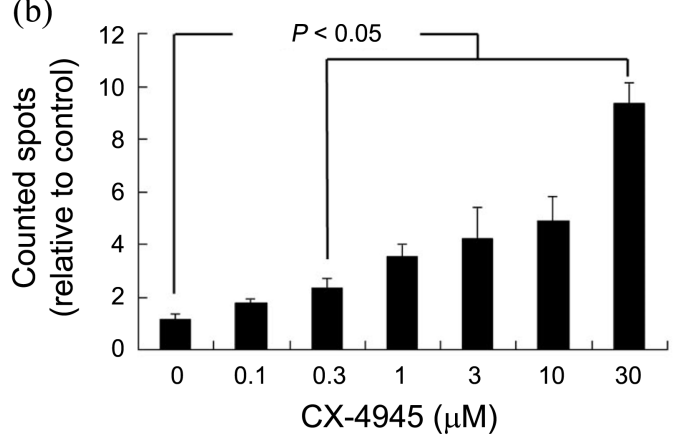

(c)

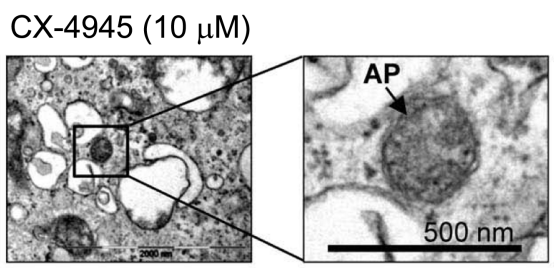

(d)

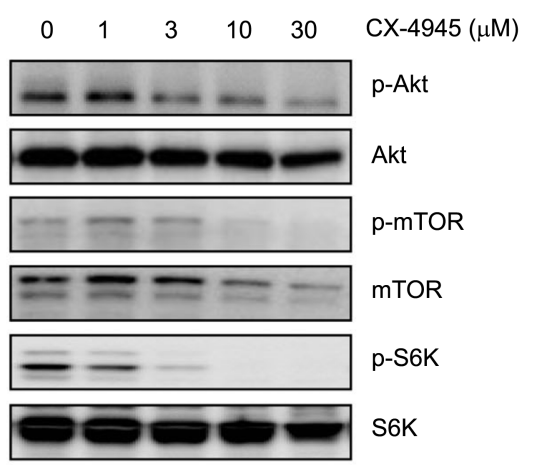

Figure 3. CX-4945 induced autophagy in HeLa cells via down-regulation of Akt/mTOR/S6K. HeLa GFP-LC3 cells were incubated with CX-4945 for $24 \mathrm{~h}$. The cells were fixed, washed, and stained with Hoechst33342 (a). GFP-LC3 spots in the cytoplasm were counted using the 'spot counting' program that comes with the Thermo Scientific Cellomics ArrayScan HCS reader (b). (c) Representative TEM image of HeLa cells after CX-4945 treatment for $24 \mathrm{~h}$. (d) HeLa cells were treated with CX-4945 for $24 \mathrm{~h}$. The expression of each protein was assessed by Western blot analysis. The detailed experimental procedures are described in the Experimental section. 
(a) $\mathrm{CX}-4945(10 \mu \mathrm{M})$

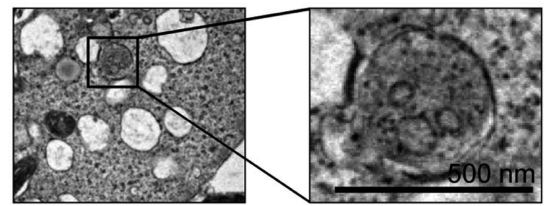

(b)

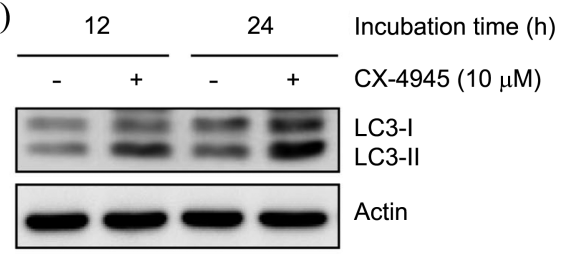

(c)

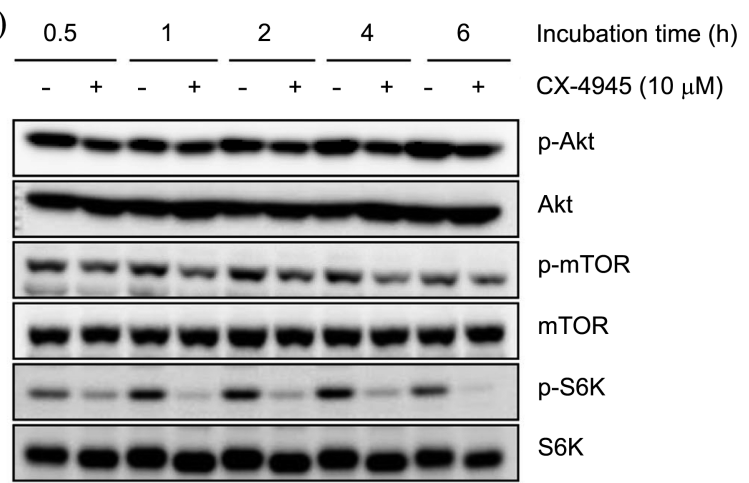

Figure 4. CX-4945 induced autophagy via down-regulation of Akt/mTOR/S6K in LNCaP cells. (a) Representative TEM image of LNCaP cells after CX-4945 treatment for $24 \mathrm{~h}$. (b) $\mathrm{LNCaP}$ cells were treated with CX-4945 for 12 and $24 \mathrm{~h}$, as indicated, and the expression of LC3-I and LC3-II was assessed by Western blot analysis. Actin was used as a loading control. (c) LNCaP cells were serum-starved for $16 \mathrm{~h}$ and then treated with CX-4945 or left untreated for $0.5,1,2,4$, and $6 \mathrm{~h}$. Protein expression was assessed by Western blot analysis. Detailed experimental procedures are described in the Material and Methods section.

LNCaP cells treated with CX-4945 formed autophagosomes (Fig. 4(a)) and showed LC3 conversion (Fig. 4(b)). CX4945 also suppressed the phosphorylation of Akt, mTOR, and S6K (Fig. 4(c)). Taken together, these results demonstrate that CX-4945 induces autophagy in both HeLa and $\mathrm{LNCaP}$ cells.

\section{Conclusion}

In conclusion, pharmacologic inhibition of CK2 by CX4945 could facilitate the autophagic cell death of cancer cells via down-regulation of the Akt-mTOR-S6K signaling cascade. CX-4945 could thus be useful as a reference compound for investigating novel autophagy-inducing agents. Further study is needed to elucidate the relationship between CK2, its specific inhibitor, and autophagy, and this knowledge could contribute to the development of autophagy-inducing agents for use as anti-cancer drugs in the future.

Acknowledgments. This work was supported by KRICT's project SI-1304, which was funded by the Ministry of Know- ledge Economy, Republic of Korea and Basic Science Research Program through the National Research Foundation of Korea (NRF) funded by the Ministry of Science ICT \& Future Planning (2014002349). The HCS analysis was the result of a study by the 'Joint Research Support Project', which was supported by the University of Science and Technology (UST). We thank Dr. Jinkyu Suk and Prof. Cheol O. Joe (Department of Biological Sciences, Korea Advanced Institute of Science and Technology, Korea) for kindly providing us with HeLa GFP-LC3 cells. We also thank Dr. Eeva-Liisa Eskelinen (Department of Biosciences, Division of Biochemistry and Biotechnology, University of Helsinki, Finland) for confirming the presence of autophagosomes in our TEM images.

\section{References}

1. Ameisen, J. C. Cell Death Differ. 2002, 9, 367.

2. Deretic, V. Trends Immunol. 2005, 26, 523.

3. Levine, B.; Yuan, J. J. Clin. Invest. 2005, 115, 2679.

4. Wang, Y.; Qin, Z. H. Acta. Pharmacol. Sin. 2013, 34, 585.

5. Scherz-Shouval, R.; Shvets, E.; Fass, E.; Shorer, H.; Gil, L.; Elazar, Z. EMBO J. 2007, 26, 1749.

6. Sui, X.; Chen, R.; Wang, Z.; Huang, Z.; Kong, N.; Zhang, M.; Han, W.; Lou, F.; Yang, J.; Zhang, Q.; Wang, X.; He, C.; Pan, H. Cell Death Res. 2013, 4, e838.

7. Fingar, D. C.; Blenis, J. Oncogene 2004, 23, 3151.

8. Laplante, M.; Sabatini, D. M. J. Cell Sci. 2009, 122, 3589.

9. Meijer, A. J.; Codogno, P. Int. J. Biochem. Cell Biol. 2004, 36, 2445.

10. Amaravadi, R. K.; Lippincott-Schwartz, J.; Wyin, X. M.; Weiss, W. A.; Takebe, N.; Timmer, W.; DiPaola, R. S.; Lotze, M. T.; White, E. Clin. Cancer Res. 2011, 17, 654.

11. Choi, K. S. Exp. Mol. Med. 2012, 44, 109.

12. Carew, J. S.; Nawrocki, S. T.; Giles, F. J.; Cleveland, J. L. Biologics 2008, 2, 201.

13. Siddiqui-Jain, A.; Drygin, D.; Streiner, N.; Chua, P.; Pierre, F.; O'Brien, S. E.; Bliesath, J.; Omori, M.; Huser, N.; Ho, C.; Proffitt, C.; Schwaebe, M. K.; Ryckman, D. M.; Rice, W. G.; Anderes, K. Cancer Res. 2010, 70, 10288.

14. Pierre, F.; Chua, P. C.; O'Brien, S. E.; Siddiqui-Jain, A.; Bourbon, P.; Haddach, M.; Michaux, J.; Nagasawa, J.; Schwaebe, M. K.; Stefan, E.; Vialettes, A.; Whitten, J. P.; Chen, T. K.; Darjania, L.; Stansfield, R.; Bliesath, J.; Drygin, D.; Ho, C.; Omori, M.; Proffitt, C.; Streiner, N.; Rice, W. G.; Ryckman, D. M.; Anderes, K. Mol. Cell Biochem. 2011, 356, 37.

15. Drygin, D.; Ho, C. B.; Omori, M.; Bliesath, J.; Proffitt, C.; Rice, R.; Siddiqui-Jain, A.; O'Brien, S.; Padgett, C.; Lim, J. K.; Anderes, K.; Rice, W. G.; Ryckman, D. Biochem. Biophys. Res. Commun. 2011, 415, 163.

16. Pierre, F.; Chua, P. C.; O'Brien, S. E.; Siddiqui-Jain, A.; Bourbon, P.; Haddach, M.; Michaux, J.; Nagasawa, J.; Schwaebe, M. K. Stefan, E.; Vialettes, A.; Whitten, J. P.; Chen, T. K.; Darjania, L.; Stansfield, R.; Anderes, K.; Bliesath, J.; Drygin, D.; Ho, C.; Omori, M.; Proffitt, C.; Streiner, N.; Trent, K.; Rice, W. G.; Ryckman, D. M. J. Med. Chem. 2011, 54, 635.

17. Kim, J.; Kim, S. H. Arch. Pharm. Res. 2012, 35, 1293.

18. Son, Y. H.; Song, J. S.; Kim, S. H.; Kim, J. Arch. Pharm. Res. 2013, 36, 840

19. Kim, J.; Kim, S. H. PLoS One 2013, 8, e74342.

20. Suk, J.; Kwak, S. S.; Lee, J. H.; Choi, J. H.; Lee, S. H.; Lee, D. H.; Byun, B.; Lee, G. H.; Joe, C. O. J. Cell Biochem. 2011, 112, 2566.

21. Lee, S. W.; Song, Y. S.; Lee, S. Y.; Yoon, Y. G.; Lee, S. H.; Park, B. S.; Yun, I.; Choi, H.; Kim, K.; Chung, W. T.; Yoo, Y. H. PLoS 
One 2011, 6, e19163.

22. Olsen, B. B.; Svenstrup, T. H.; Guerra, B. Int. J. Oncol. 2012, 41, 1967.

23. Hurren, R.; Beheshti Zavareh, R.; Dalili, S.; Wood, T.; Rose, D.; Chang, H.; Jamal, N.; Messner, H.; Batey, R. A.; Schimmer, A. D.
Apoptosis 2008, 13, 748.

24. Ryu, B. J.; Baek, S. H.; Kim, J.; Bae, S. J.; Chang, S. Y.; Heo, J. N.; Lee, H.; Lee, S. Y.; Kim, S. H. Bioorg. Med. Chem. Lett. 2012, 22,5470 . 